\title{
The Phonon-Assisted Radiative Recombination of Excitons Confined in InAs Quantum Dashes
}

\begin{abstract}
Ł. Dusanowski*, A. Musiae, G. SęK and P. Machnikowski
Institute of Physics, Wrocław University of Technology, Wybrzeże Wyspiańskiego 27, 50-370 Wrocław, Poland

In this report we have investigated theoretically the phonon-assisted recombination process of excitons confined in strongly elongated semiconductor nanostructures, called quantum dashes. Interaction with phonon bath leads to the occurrence of phonon-assisted recombination, which in the case of acoustic phonons is manifested in the optical spectra as a deviation of the homogeneously broadened emission line shape from expected Lorentzian profile via occurrence of the so-called phonon sidebands. Hereby, we have modeled the influence of the quantum dash geometry on this spectral feature proving pronounced suppression of phonon-induced decoherence for strongly elongated nanostructures. Furthermore, the importance of different phonon coupling mechanisms has been evaluated and the spectral diffusion effects, unavoidable in the time-integrated photoluminescence experiments, have been accounted for.
\end{abstract}

DOI: $10.12693 /$ APhysPolA.124.813

PACS: $78.67 . \mathrm{Hc}, 63.20 . \mathrm{kk}$

\section{Introduction}

The properties of a quantum system that interacts with the environment in a thermodynamically irreversible way are affected in various ways by the environmental influence. In the case of epitaxial nanostructures, the main effect is due to interaction with phonons, which manifests itself in the line shape observed in optical absorption or emission. One of the experimentally observable phonon-induced effects is the appearance of phonon sidebands which results from exciton generation or recombination accompanied by phonon absorption or emission, so that the photon energy is shifted by the energy of the involved phonons [1-3].

In the case of emission lines, this effect has already been investigated experimentally in symmetric quantum dots (QDs), where by analyzing the exciton line shape at different temperatures a strong deviation from expected Lorentzian profile has been observed by appearance of low-energy acoustic-phonon sidebands [4]. However, no study has yet been performed on anisotropic objects, geometry of which could strongly influence the strength of the exciton-acoustic phonon coupling.

In this report we have investigated theoretically the phonon-assisted recombination process of excitons confined in such strongly elongated semiconductor nanostructures, called quantum dashes (QDashes) [5]. In our calculations we have included electron-hole correlation effects as well as coupling of excitons with acoustic-phonons by two mechanisms: (i) deformation potential (DP) and (ii) piezoelectric field (PE). We have shown which effect dominates and analyzed the changes of the exciton homogeneous broadening as a function of phonon

*corresponding author; e-mail: lukasz.dusanowski@pwr.wroc.pl mode occupations (temperature) for different QDash geometries and initial (low-temperature) inhomogeneous broadenings of the exciton line, mimicking the spectral diffusion (SD) effects. All the calculations were performed for InAs/InP based structures which, besides the possibility of tailoring the dash dimensions in a wide range [5], are especially interesting due to the emission at telecommunication wavelengths [6] and selective polarization properties, making them favorable for some novel optoelectronic applications like single photon sources or microlasers that could be easily integrated with the optical fibers $[7,8]$.

\section{The model}

Due to its significant anisotropy, the confining potential is approximated by an infinite well in the direction of the elongation (and as a result much weaker quantum confinement) whereas the dynamics in the cross-sectional directions is restricted to the ground state and a $2 \mathrm{D}$ harmonic oscillator potential is assumed.

In that case single-carrier electron (e) and hole (h) states can be described analytically

$$
\psi_{n}^{(\mathrm{e} / \mathrm{h})}(\boldsymbol{r})=\sqrt{\frac{2}{\pi l_{z} l_{y} L}} \exp \left(-\frac{y^{2}}{2 l_{y}^{2}}-\frac{z^{2}}{2 l_{z}^{2}}\right) \sin \left(\frac{n \pi x}{L}\right),
$$

where $l_{y}, l_{z}$ are localization widths in the cross-section, $L$ is the well width in the direction of the elongation (QDash length) and $n$ is a principal quantum number. Parameters $l_{y}$ and $l_{z}$ correspond to the characteristic QDash width $D$ and height $H\left(D=2 l_{y} \sqrt{2 \ln 2}\right.$ and analogically for $H$ ). Based on these single-particle wave functions one can construct the product basis for the excitonic states and diagonalize the Hamiltonian of the system which has the form

$$
H_{0}=\sum_{i} E_{i}^{(\mathrm{e})} a_{i}^{\dagger} a_{i}+\sum_{i} E_{i}^{(\mathrm{h})} h_{i}^{\dagger} h_{i}+\sum_{i j k l} V_{i j k l} a_{i}^{\dagger} h_{j}^{\dagger} h_{k} a_{l},
$$


where $a_{i}^{\dagger}, a_{i}$ and $h_{i}^{\dagger}, h_{i}$ are electron and hole creation and annihilation operators. The first two terms describe the free carrier Hamiltonian, where $E_{n}^{(\mathrm{e})}$ are electron (hole) energies equal to

$$
E_{n}^{(\mathrm{e} / \mathrm{h})}=E_{0}^{(\mathrm{e} / \mathrm{h})}+\frac{\hbar^{2} \pi^{2} n^{2}}{m_{\mathrm{e} / \mathrm{h}}^{*} 2 L^{2}},
$$

expressed by the sum of the harmonic oscillator ground state energy $E_{0}^{(\mathrm{e} / \mathrm{h})}$ and the energy of infinite well quantized levels. The third term is the interaction part of the Hamiltonian in which $V_{i j k l}$ are the matrix elements of the electron-hole interaction

$$
\begin{array}{r}
V_{i j k l}=-\int \mathrm{d}^{3} r_{\mathrm{e}} \int \mathrm{d}^{3} r_{\mathrm{h}} \psi_{i}^{(\mathrm{e}) *}\left(\boldsymbol{r}_{\mathrm{e}}\right) \psi_{j}^{(\mathrm{h}) *}\left(\boldsymbol{r}_{\mathrm{h}}\right) \\
\times\left(\frac{e^{2}}{4 \pi \varepsilon_{\mathrm{s}} \varepsilon_{0}} \frac{1}{\left|\boldsymbol{r}_{\mathrm{e}}-\boldsymbol{r}_{\mathrm{h}}\right|}\right) \psi_{k}^{(\mathrm{h})}\left(\boldsymbol{r}_{\mathrm{h}}\right) \psi_{l}^{(\mathrm{e})}\left(\boldsymbol{r}_{\mathrm{e}}\right),
\end{array}
$$

where $\varepsilon_{0}$ is the vacuum permittivity and $\varepsilon_{\mathrm{s}}$ is the static relative dielectric constant.

Upon diagonalization of the Hamiltonian we derived the coefficients $C_{n m}$ for the expansion of the exciton ground state in the single particle states basis, where $|0\rangle$ is the crystal ground state,

$$
|X\rangle=\sum_{i j} C_{i j} a_{i}^{\dagger} h_{j}^{\dagger}|0\rangle,
$$

which were then used for the calculations of phonon spectral densities and phonon-assisted exciton emission spectra as a function of temperature.

At this point, the coupling between electrons and acoustic phonons is included in the Hamiltonian of the system

$$
H=H_{0}+H_{\mathrm{ph}}+H_{\mathrm{int}},
$$

where $H_{0}$ describes carriers subsystem and $H_{\mathrm{ph}}$ is the free phonon Hamiltonian

$$
H_{\mathrm{ph}}=\sum_{\boldsymbol{k}, s} \hbar \omega_{\boldsymbol{k}} b_{\boldsymbol{k}, s}^{\dagger} b_{\boldsymbol{k}, s}
$$

where $b_{\boldsymbol{k}, s}^{\dagger}, b_{\boldsymbol{k}, s}$ are creation and annihilation operators for a phonon in the mode $s$ with the wave vector $k$ $(s=1$, t denotes the LA and TA phonon branches, respectively). The last term corresponds to the excitonphonon interaction, $H_{\text {int }}=H_{\text {int }}^{(\mathrm{e})}+H_{\text {int }}^{(\mathrm{h})}$, with

$$
H_{\mathrm{int}}^{(\mathrm{e})}=\sum_{n n^{\prime}, \boldsymbol{k}, s} F_{n n^{\prime}, \boldsymbol{k}, s}^{(\mathrm{e})}\left(b_{\boldsymbol{k}, s}^{\dagger}+b_{-\mathbf{k}, s}\right) a_{n}^{\dagger} a_{n^{\prime}}
$$

and analogous for holes, where $F_{n n^{\prime}, \boldsymbol{k}, s}=F_{n n^{\prime},-\boldsymbol{k}, s}^{*}$ are coupling constants. Since only long-wavelength phonons are effectively coupled to carriers confined in a QDash [9] we will always assume linear and isotropic dispersion for acoustic phonons $\omega_{k}=c_{\mathrm{s}} k$, where $c_{\mathrm{s}}$ is speed of sound. The phonon spectral densities defining spectral properties of the crystal lattice are

$$
R(\omega)=\sum_{\boldsymbol{k}, s}\left|\frac{F_{\boldsymbol{k}, s}^{(X)}}{\hbar c_{\mathrm{s}} k}\right|^{2}\left[n_{\mathrm{B}}(\omega) \delta\left(\omega+c_{\mathrm{s}} k\right)\right.
$$

$$
\begin{aligned}
& \left.+\left(n_{\mathrm{B}}(\omega)+1\right) \delta\left(\omega-c_{\mathrm{s}} k\right)\right] \\
& =\frac{1}{(2 \pi)^{3}} \sum_{\mathrm{s}} \int \mathrm{d}^{3} k\left|\frac{F_{\boldsymbol{k}, s}^{(X)}}{\hbar c_{\mathrm{s}} k}\right|^{2}\left[n_{\mathrm{B}}(\omega) \delta\left(\omega+c_{\mathrm{s}} k\right)\right. \\
& \left.+\left(n_{\mathrm{B}}(\omega)+1\right) \delta\left(\omega-c_{\mathrm{S}} k\right)\right],
\end{aligned}
$$

where $n_{\mathrm{B}}(\omega)$ is the Bose distribution function describing the influence of the temperature on the phonon modes occupation, $F_{\boldsymbol{k}, s}^{(X)}$ is the excitonic phonon coupling constant

$$
\begin{aligned}
& F_{\boldsymbol{k}, s}^{(X)}=\sum_{i j k} C_{i k}^{*} C_{j k} F_{i j, \boldsymbol{k}, s}^{(\mathrm{e})}+\sum_{i j k} C_{k i}^{*} C_{k j} F_{i j, \boldsymbol{k}, s}^{(\mathrm{h})} \\
& F_{i j, \boldsymbol{k}, s}^{(\mathrm{e} / \mathrm{h})}=F_{i j, \boldsymbol{k}, s}^{(\mathrm{e} / \mathrm{h}) \mathrm{PE}}+F_{i j, \boldsymbol{k}, s}^{(\mathrm{e} / \mathrm{h}) \mathrm{DP}},
\end{aligned}
$$

where $F_{i j, \boldsymbol{k}, s}^{(\mathrm{e} / \mathrm{h} \mathrm{PE}}$ and $F_{i j, \boldsymbol{k}, s}^{(\mathrm{e} / \mathrm{h}) \mathrm{DP}}$ are electron and hole coupling constants for PE and DP mechanisms, respectively, calculated as in Ref. [9]. Based on the standard results for the independent boson model [3, 4], the emission spectrum for the recombination of a confined exciton is modeled as

$$
\begin{aligned}
& f(\omega)=\frac{1}{\sqrt{2 \pi} \sigma} \exp \left(R_{\infty}-\frac{1}{2}\left(\frac{\omega^{2}}{\sigma^{2}}\right)\right) \\
& +\frac{1}{2 \pi} \int \mathrm{d} t \exp (\mathrm{i} \omega t) \exp \left(-\frac{1}{2} \sigma^{2} t^{2}\right) \\
& \times[\exp (\tilde{R}(t))-1],
\end{aligned}
$$

where $R_{\infty}=-R(0), \tilde{R}(t)$ is the Fourier transform of $R(\omega)$ and $\sigma$ describes the initial (low-temperature) width of the central exciton line, which is assumed to be of a Gaussian profile. One has to notice that exciton should in general have a Lorentzian line shape. In an experiment however, when the time-integrated spectra are collected, limited setup resolution broadens inhomogeneously the exciton line and the spectral diffusion effects [10] lead to fluctuations of exciton emission energy, so that finally a Gaussian profile is observed. Moreover, in our modeling we assume that this initial inhomogeneous broadening is primarily caused by spectral diffusion, thus initial line width will be later on denoted as SD.

\section{Results}

In this section we present results of theoretical modeling of the exciton-acoustic phonon coupling effect on the inhomogeneous emission line shape of the single exciton confined in a QDash. The modeled structures are quantum dot-like objects made of InAs on InP (001) substrate, elongated in [1-10] direction, with sizes varying from $50 \times 11 \times 2 \mathrm{~nm}^{3}$ to $300 \times 25 \times 4.5 \mathrm{~nm}^{3}$, i.e. in the experimentally relevant range of geometries [5].

In Fig. 1 emission spectra for a typical In As/InP structure $\left(4.5 \times 25 \times 150 \mathrm{~nm}^{3}\right)$ is presented in the temperature range from 5 to $200 \mathrm{~K}$. Initial width (full width at half 
maximum - FWHM) of the exciton line, has been set to $0.1 \mathrm{meV}$ (typical broadening value observed experimentally for this type of nanostructures). For clarity, the emission lines for different temperatures are shifted horizontally. One can clearly observe that with increasing temperature the lines become broader and start to differ significantly from the initial Gaussian profile, especially at their bottom where the phonon-related sidebands start to appear. At high temperatures the spectrum is already fully dominated by phonon-assisted recombination.

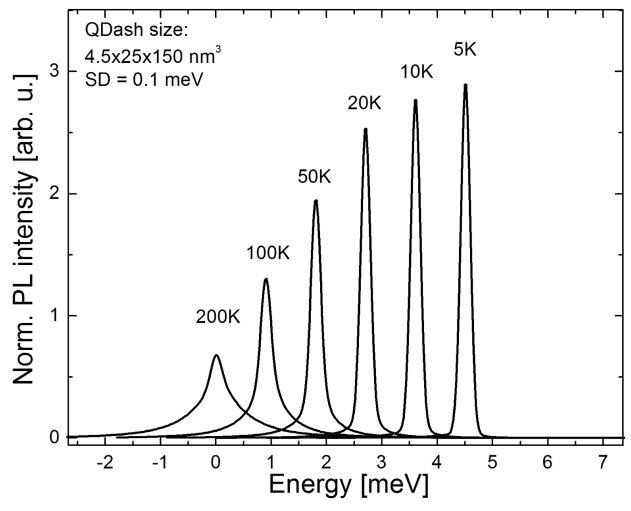

Fig. 1. Calculated single exciton PL spectra of $4.5 \times$ $25 \times 150 \mathrm{~nm}^{3}$ InAs QDash, normalized to the integrated intensity for various temperatures. For clarity, the energy position of emission lines are shifted horizontally.

To compare the strength of different exciton-phonon coupling mechanisms, phonon spectral densities (representing the oscillator strength of the phonon-assisted recombination of excitons) have been calculated (Fig. 2) for DP (a) and PE (b) coupling separately. It turns out that the PE coupling is around 3 orders of magnitude weaker than the DP one. This is mainly because of a small spatial separation of electron and hole despite a large QDash volume. In both cases the emission spectra are asymmetric at low temperatures due to low phonon concentration favoring processes with the emission of phonons. When temperature increases it tends to symmetrize and at temperatures high compared to the width of the phonon feature no asymmetry is observed, because both emission and absorption events are of the same importance.

Finally, we have analyzed the influence of QDash geometry on phonon-induced exciton decoherence. In Fig. 3 the temperature dependence of the exciton emission line FWHM for two lengths of a QDash (a) $L=100 \mathrm{~nm}$ and (b) $L=150 \mathrm{~nm}$ and set of widths $D$ ranging from 11 to $27 \mathrm{~nm}$ (with $H=D / 5.5$ after Ref. [5]) is presented. At low temperatures slow and linear rise of FWHM up to $100 \mathrm{~K}$ for $100 \mathrm{~nm}$ ( $150 \mathrm{~K}$ for $150 \mathrm{~nm}$ ) QDash can be observed. Above this critical temperature it increases rapidly. The slope of that increase slightly depends on the cross-sectional dimension whereas the critical temperature is insensitive to this parameter. In parts (c) and (d) analogical dependences for the spectral diffusion in the range of 0.1 to $0.5 \mathrm{meV}$ are presented. Increasing

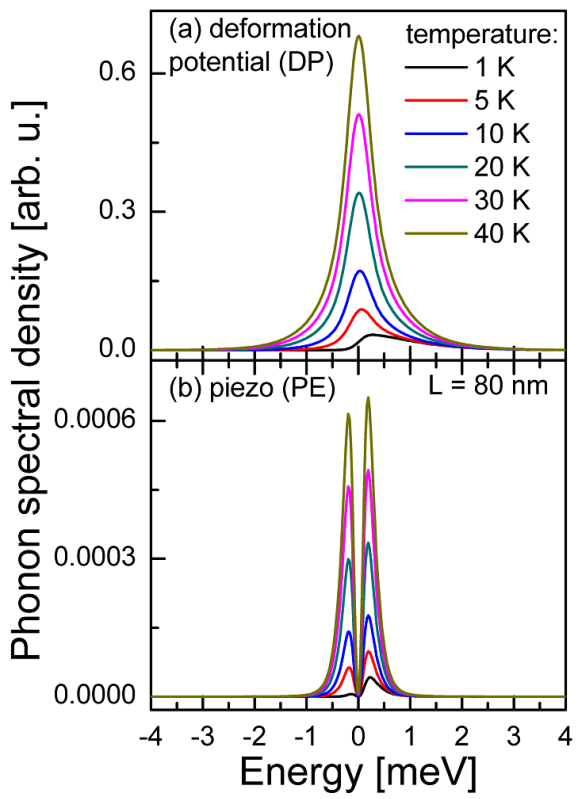

Fig. 2. Calculated phonon spectral densities for $4.5 \times$ $25 \times 80 \mathrm{~nm}^{3}$ InAs QDash at various temperatures, assuming exciton interaction with acoustic phonons via (a) deformation potential, and (b) piezoelectric effect.

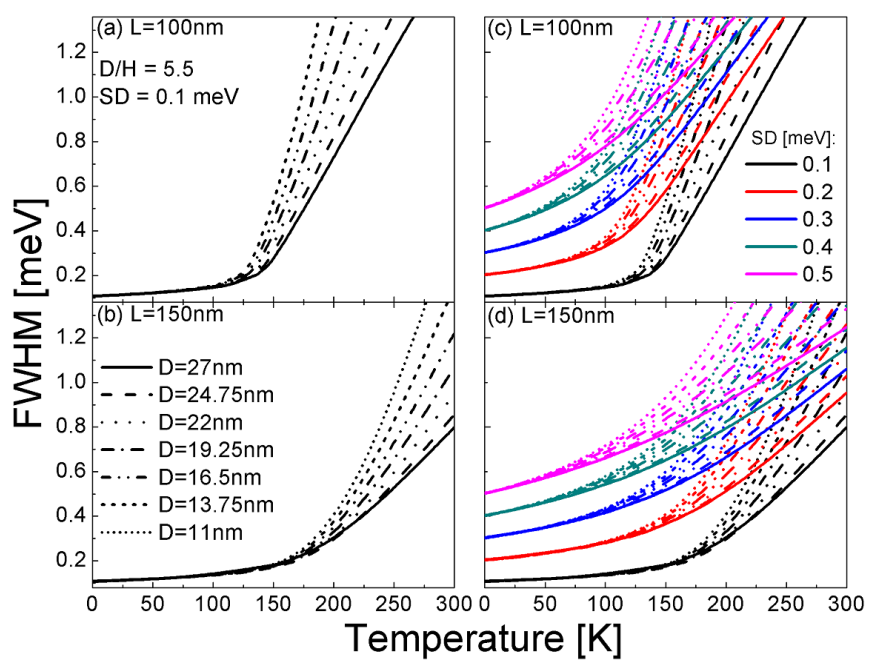

Fig. 3. Calculated temperature dependence of the exciton emission line FWHM for two lengths of QDash (a) $L=100 \mathrm{~nm}$ and (b) $L=150 \mathrm{~nm}$ and set of widths $D$ ranging from 11 to $27 \mathrm{~nm}$ (with $H=D / 5.5$ ). In parts (c) and (d) analogical dependences for spectral diffusions (SD) in the range of 0.1 to $0.5 \mathrm{meV}$ are presented.

the SD strongly changes the FWHM dependence on temperature. For low SD two characteristic regimes can be distinguished, i.e. slow and fast increase of FWHM. For larger SD (>0.3 meV), no such dependence is present and fast smooth increase of FWHM is observed instead. The elongation of a QDash does not change the picture qualitatively, but the critical temperature reaches higher values. 


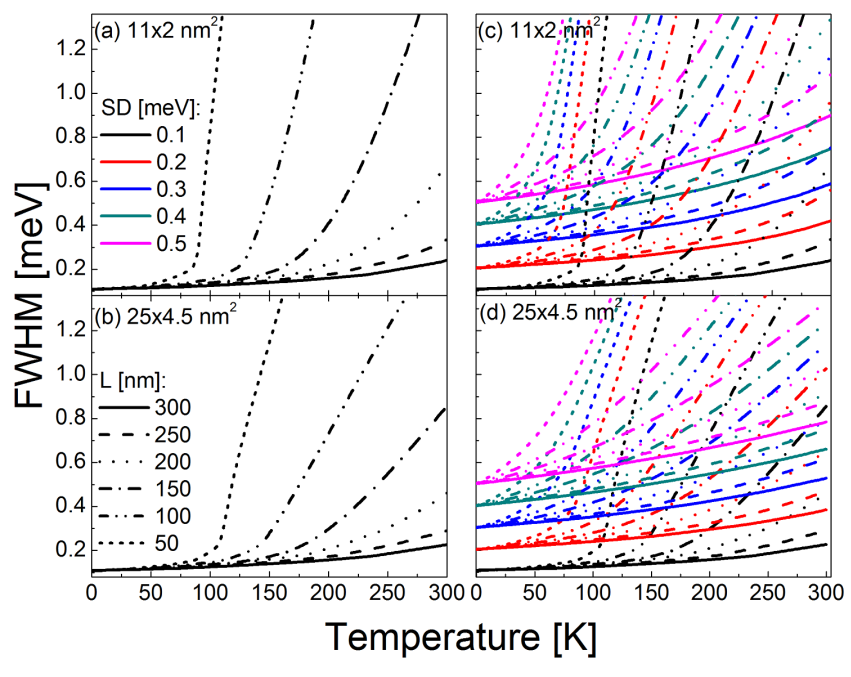

Fig. 4. Calculated temperature dependence of the exciton emission line FWHM for two cross-sectional dimensions of QDash (a) $11 \times 2 \mathrm{~nm}^{2}$ and (b) $25 \times 4.5 \mathrm{~nm}^{2}$ and set of lengths $L$ ranging from 50 to $300 \mathrm{~nm}$. In parts (c) and (d) analogical dependences for spectral diffusion (SD) in the range of 0.1 to $0.5 \mathrm{meV}$ are presented.

This observation is even more pronounced in Fig. 4 where the temperature dependences of exciton line FWHM are presented for two cross-sectional dimensions of the QDash (a) $11 \times 2 \mathrm{~nm}^{2}$ and (b) $25 \times 4.5 \mathrm{~nm}^{2}$ and various QDash lengths $L$ ranging from 50 to $300 \mathrm{~nm}$. When the QDash length is increased from 50 to $150 \mathrm{~nm}$, the critical temperature shifts from 100 to 200 K. Further increase of the QDash length ( $>200 \mathrm{~nm}$ ) extends the range of temperatures at which the excitonic line shape is only slightly affected by the phonon-coupling. It means that the elongation of the structure should in general decrease the strength of the acoustic phonon-exciton coupling and, therefore, also the decoherence of an exciton state confined in such a strongly elongated object should be strongly suppressed even up to room temperature.

\section{Conclusions}

We have studied theoretically the phonon-assisted recombination of excitons confined in InAs/InP semicon- ductor quantum dashes. We have found that phonons couple to the exciton practically only by deformation potential mechanism, so that piezoelectric contribution is negligible. Analysis of the QDash geometry influence on the FWHM temperature dependence revealed rather weak impact of the cross-sectional dimensions and the crucial role of the QDash length. Elongation of the dashes should in general suppress the interaction with acoustic phonons. QDashes as long as 200-300 nm are predicted to enable room temperature emission with negligible contribution of phonon-assisted transitions.

\section{Acknowledgments}

The authors acknowledge the financial support from the National Science Centre of Poland within grant No. 2011/02/A/ST3/00152 and the fellowship co-financed by European Union within European Social Fund.

\section{References}

[1] K. Huang, A. Rhys, Proc. R. Soc. Lond. A 204, 406 (1950).

[2] C.B. Duke, G.D. Mahan, Phys. Rev. 139, A1965 (1965).

[3] G.D. Mahan, Many-Particle Physics, Plenum, New York 1990

[4] L. Besombes, K. Kheng, L. Marsal, H. Mariette, Phys. Rev. B 63, 155307 (2001).

[5] A. Sauerwald, T. Kümmell, G. Bacher, A. Somers, R. Schwertberger, J.P. Reithmaier, A. Forchel, Appl. Phys. Lett. 86, 253112 (2005).

[6] J.P. Reithmaier, G. Eisenstein, A. Forchel, Proc. IEEE 95, 1779 (2007).

[7] T. Miyazawa, K. Takemoto, Y. Sakuma, S. Hirose, T. Usuki, N. Yokoyama, M. Takatsu, Y. Arakawa, Jpn. J. Appl. Phys. 44, L620 (2005).

[8] N. Chauvin, P. Nedel, C. Seassal, B. Ben Bakir, X. Letartre, M. Gendry, A. Fiore, P. Viktorovitch, Phys. Rev. B 80, 045315 (2009).

[9] Quantum Dots: Research Developments, Ed. P.A. Ling, Nova Science, New York 2005, p. 47.

[10] J. Seufert, R. Weigand, G. Bacher, T. Kümmell, A. Forchel, K. Leonardi, D. Hommel, Appl. Phys. Lett. 76, 1872 (2000). 